(C) Revista de Matemática: Teoría y Aplicaciones 2022 29(1) : 39-52

CIMPA - UCR ISSN: 1409-2433 (PRINT), 2215-3373 (ONLINE)

DOI: https://doi.org/10.15517/rmta.v29i1.45440

\title{
APROXIMACIÓN TRIGONOMÉTRICA EN ESPACIOS LIPSCHITZ
}

\section{TRIGONOMETRIC APPROXIMATION IN LIPSCHITZ SPACES}

\author{
GERARDo MARTÍNEZ-GUZMÁN* \\ MARÍA BEATRÍZ BERNÁBE LORANCA ${ }^{\dagger}$ \\ MARIANO LARIOS GÓMEZ JORGE RuIZ VANOYE $^{\S}$
}

Received: 4/Mar/2021; Revised: 11/Nov/2021;

Accepted: 8/Dec/2021

Revista de Matemática: Teoría y Aplicaciones is licensed under a Creative Commons Attribution-NonCommercial-ShareAlike 4.0 International License.

http://creativecommons.org/licenses/by-nc-sa/4.0/

(c) (i) (2) (2)

BY NC SA

*Benemérita Universidad Autónoma de Puebla, Facultad de Ciencias de la Computación, Puebla, México. E-Mail: gmartinez54@hotmail.com

${ }_{\dagger}^{\dagger}$ Benemérita Universidad Autónoma de Puebla, Facultad de Ciencias de la Computación, Puebla, México. E-Mail: beatriz.bernabe@gmail.com

${ }^{\ddagger}$ Benemérita Universidad Autónoma de Puebla, Facultad de Ciencias de la Computación, Puebla, México. E-Mail: mariano.larios@correo.buap.mx

${ }^{\S}$ Universidad Politécnica de Pachuca, Departamento de Ingeniería de Software, Pachuca, México. E-Mail: jorge@ ruizvanoye.com 


\begin{abstract}
La aproximación por polinomios trigonométricos generalizados para funciones de Lipschitz, definidas en ciertos grupos depende de algunas propiedades de la métrica definida en el grupo. Métricas donde esta aproximación es posible son llamadas Lipschitz compatibles. En este trabajo damos para cierta clase de grupos, condiciones donde las métricas Lipschitz compatibles son acotadamente equivalentes, es decir, generan el mismo espacio de Lipschitz. En particular, para el grupo multiplicativo de números complejos con norma uno las condiciones son necesarias y suficientes para que las métricas Lipschitz compatibles sean acotadamente equivalentes.
\end{abstract}

Keywords: espacios de Lipschitz; métricas invariantes; polinomios trigonométricos; grupos topológicos; espacio dual.

\title{
Resumen
}

The approximation by generalized trigonometric polynomials for Lipschitz defined functions in certain groups depends on some properties of the group defined metric. Metrics which allow this approximation are called Lipschitz compatible. In this work we give for certain class of groups, conditions under which Lipschitz compatible metrics are boundedly equivalent, i.e., they generate the same Lipschitz space. In particular, for the multiplicative group of modulus one complex numbers the conditions are necessary and sufficient for the compatible Lipschitz metrics to be boundedly equivalent.

Keywords: Lipschitz spaces; invariant metrics; trigonometric polynomials; topological groups; dual space.

Mathematics Subject Classification: 51F30, 32F45, 41A10, 28C10, 47L50.

\section{Introducción}

Sea $T$ el grupo de números complejos con norma igual a uno, con el producto de números complejos como operación. Un carácter, de un grupo abeliano localmente compacto, es una función continua $\chi: \mathrm{G} \rightarrow \mathrm{T}$, tal que, para todo $x, y \in$ $G$ se tiene que $\chi(x y)=\chi(x) \chi(y)$. Esto es, $\chi$ es un morfismo de grupos. Los caracteres de $G$ forman ellos mismos un grupo abeliano bajo la multiplicación puntual, esto es, para todo $x \in G,\left(\chi_{1}+\chi_{2}\right)(x)=\chi_{1}(x) \chi_{2}(x)$.

En el grupo de caracteres de un grupo abeliano localmente compacto, se define la topología compacta abierta que lo convierte en un grupo topológico localmente compacto. Este grupo es llamado el grupo dual de $G$ y se denota por $\Gamma$ [4], [5]. 
Definición 1 Sea $G$ un grupo topológico abeliano, una métrica d en $G$, se dice que es invariante si $\forall g, h, k \in G, d(k g, k h)=d(g, h)$

Definición 2 Dos métricas $d_{1}$ y $d_{2}$ en $G$ son llamadas acotadamente equivalentes si y sólo si existen números positivos $A$ y $B$, tales que, para cualesquiera $x, y \in G$,

$$
A d_{1}(x, y) \leq d_{2}(x, y) \leq B d_{1}(x, y)
$$

Las métricas $d_{1}$ y $d_{2}$ se dicen equivalentes si y sólo si el mapeo identidad $I d:\left(G, d_{1}\right) \rightarrow\left(G, d_{2}\right)$ es un homeomorfismo. Es decir, las topologías inducidas por las métricas $d_{1}$ y $d_{2}$ coinciden. Por supuesto que la equivalencia acotada implica la equivalencia. Aplicaciones de métricas invariantes en grupos pueden encontrarse en [1], [6], [8].

Teorema 1 Si un grupo $G$ es compacto y metrizable, entonces su grupo dual $\Gamma$ es discreto y numerable [2].

\section{Espacios de Lipschitz en grupos topológicos}

Definiremos los espacios de Lipschitz en un grupo topológico localmente compacto, con una métrica invariante cuya topología generada coincide con la topología del grupo [3], [9].

Definición 3 Sea $G$ un grupo abeliano localmente compacto con una métrica d invariante y sean $f \in C(G)$ conjunto de funciones continuas en $G$ y $0<\alpha \leq 1$, definimos:

$$
\theta_{\alpha}(f):=\sup _{\delta>0}\left\{\frac{|f(x)-f(y)|}{d(x, y)^{\alpha}}: 0<d(x, y) \leq \delta\right\}
$$

Entonces los espacios de Lipschitz se definen como:

$$
\operatorname{Lip}_{\alpha}(G):=\left\{f \in C(G) / \theta_{\alpha}(f)<\infty\right\} .
$$

El espacio de $\operatorname{Lip}_{\alpha}(G)$ es un espacio normado con la norma [2], definida como:

$$
\|f\|_{\alpha}:=\max \left(\|f\|_{\infty}, \theta_{\alpha}(f)\right) .
$$

Cualquier función de la forma

$$
p(x)=\sum_{j=1}^{k} a_{j} \gamma_{j}(x) .
$$


donde $\gamma_{j} \in \Gamma, a_{j} \in \mathbb{C}$ es llamado un polinomio trigonométrico generalizado en $G$. Es conocido que si $G$ es compacto entonces el conjunto $P(G)$, de todos los polinomios trigonométricos generalizados, es una $\mathbb{C}$-álgebra y separa puntos. Así, por el teorema de Stone Weierstrass, $P(G)$ es denso en $C(G)$ [5]. Estamos particularmente interesados en estudiar la aproximación de funciones que están en $\operatorname{Lip}_{1}(G)$ por polinomios trigonométricos generalizados en $G$. Es decir, aproximación por funciones del tipo (1). En el caso $G=T$, los polinomios trigonométricos generalizados son precisamente los polinomios trigonométricos clásicos, pues $\Gamma$ se identifica con $\mathbb{Z}$ y todo carácter es de la forma $\gamma(x)=e^{i n x}$ con $n \in \mathbb{Z}$. Tomando en consideración lo anterior, consideramos en el resto del trabajo que el grupo $G$ es topológicamente isomorfo a $T^{r}$ para algún $r \geq 1$.

Estamos interesados en las métricas que generan los mismos espacios de Lipschitz, lo que da lugar a citar el siguiente teorema:

Teorema 2 Considere dos métricas en G. Los espacios de Lipschitz generados por estas dos métricas coinciden si y solo si las métricas son acotadamente equivalentes [7].

Las métricas equivalentes en el sentido topológico, que son invariantes por traslación pero que no son equivalentemente acotadas como métricas, implican en general la presencia de diferentes espacios de Lipschitz. Es decir, los espacios de Lipschitz no están asociados exactamente con la estructura topológica del grupo sino con las métricas que lo definen.

Lo anterior nos plantea el problema de encontrar de alguna manera una distancia $d$ dentro de un grupo de distancias en $G$, que sean invariantes por traslación y que nos garantice el mismo espacio de Lipschitz, donde podamos utilizar los polinomios generalizados en los problemas de aproximación, de manera similar a lo que ocurre con la aproximación trigonométrica usual.

Definición 4 Decimos que el grupo dual $\Gamma$ es finitamente generado, si existe un conjunto de caracteres $B=\left\{\chi_{1}, \chi_{2}, \ldots, \chi_{r}\right\} \subseteq \Gamma$ tal que si $\phi \in \Gamma$, entonces es de la forma

$$
\phi=\sum_{i=1}^{r} n_{i} \chi_{i}, n_{i} \in \mathbb{Z} .
$$

Si queremos trabajar la aproximación por polinomios trigonométricos generalizados en los espacios de Lipschitz la métrica debe satisfacer ciertas condiciones, por lo cual damos la siguiente definición. 
Definición 5 Sea d una métrica en $G \times G$ que define la topología de $G$. Entonces decimos que d es Lipschitz compatible si satisface:

1. des una métrica invariante en $G$.

2. $\Gamma \subseteq \operatorname{Lip}_{1}(G)$ con respecto a la métrica $d$.

Teorema 3 Sea $G$ un grupo topológicamente isomorfo a $T^{r}$ con $r \geq 1$ cuyo dual $\Gamma$ es generado por $B=\left\{\chi_{1}, \chi_{2}, \ldots, \chi_{r}\right\}$, según [5]. Entonces

1. la función

$$
d_{B}(x, y):=\Sigma_{1 \leq i \leq r}\left|\chi_{i}(x)-\chi_{i}(y)\right|,
$$

es una métrica invariante que induce la topología de $G$.

2. Si $B^{1}$ es otro generador finito de $\Gamma$ y definimos $d_{B^{1}}$ análogamente a $d_{B}$, estas distancias resultan acotadamente equivalentes.

3. La métrica $d_{B}$ en $G$ es Lipschitz compatible.

4. Si d es cualquier métrica Lipschitz compatible en $G$, existe una constante $C$ tal que $d_{B} \leq C d$.

Demostración. 1. Como $B=\left\{\chi_{1}, \chi_{2}, \ldots, \chi_{r}\right\}$ es generador de $\Gamma$, las funciones $\left(\chi_{i}\right)_{1 \leq i \leq r}$ separan puntos, de donde si $x \neq y$, entonces $d_{B}(x, y) \neq 0$. Los demás axiomas de métrica se verifican sin dificultad. Por otro lado, de la continuidad de los caracteres $\chi_{i}$ se deduce la continuidad de la inyección $G \rightarrow\left(G, d_{B}\right)$ por lo que es un homeomorfismo. Además, la métrica $d_{B}$ es invariante, ya que,

$$
\left|\chi_{i}(x z)-\chi_{i}(y z)\right|=\left|\chi_{i}(x)-\chi_{i}(y)\right|
$$

2. Primero demostraremos que para cualesquiera $x_{j}, y_{j} \in T$ y $n_{j} \in \mathbb{Z}$, existe una constante $C \geq 0$, que depende de los $n_{j}$, pero no de los elementos $x_{j}, y_{j}$, tal que

$$
\left|\prod_{1 \leq j \leq r} x_{j}^{n_{j}}-\prod_{1 \leq j \leq r} y_{j}^{n_{j}}\right| \leq C \sum_{1 \leq j \leq r}\left|x_{j}-y_{j}\right| .
$$

En efecto, considere $a, b, c$ y $d$ números complejos en T y sean $n, m \in \mathbb{Z}$, entonces los números $a^{n}, c^{n}, b^{m}$ y $d^{m}$ pertenece a T, en el caso que $n$ o $m$ sean negativos usando la igualdad $a^{n}=(\bar{a})^{-n}$, además se cumple que $|\bar{a}-\bar{c}|=|a-c|$ y $|\bar{b}-\bar{d}|=|b-d|$. Entonces,

$$
\begin{aligned}
\left|a^{n} b^{m}-c^{n} d^{m}\right| & =\left|a^{n} b^{m}+b^{m} c^{n}-b^{m} c^{n}-c^{n} d^{m}\right| \\
& =\left|b^{m}\left(a^{n}-c^{n}\right)+c^{n}\left(b^{m}-d^{m}\right)\right| \\
& \leq\left|a^{n}-c^{n}\right|+\left|b^{m}-d^{m}\right| .
\end{aligned}
$$


Así obtenemos la desigualdad

$$
\left|a^{n} b^{m}-c^{n} d^{m}\right| \leq\left|a^{n}-c^{n}\right|+\left|b^{m}-d^{m}\right| .
$$

Pero, sabemos que

$$
a^{n}-c^{n}=(a-c)\left(a^{n-1}+a^{n-2} c+\cdots+c^{n-1}\right) .
$$

Entonces tenemos

$$
\left|a^{n}-c^{n}\right| \leq n|a-c| y\left|b^{m}-d^{m}\right| \leq m|b-d| .
$$

Así, de (5) obtenemos

$$
\left|a^{n} b^{m}-c^{n} d^{m}\right| \leq n|a-c|+m|b-d| .
$$

Usando inducción matemática, y la desigualdad (6), tenemos,

$$
\left|\prod_{1 \leq j \leq r} x_{j}^{n_{j}}-\prod_{1 \leq j \leq r} y_{j}^{n_{j}}\right| \leq \sum_{1 \leq j \leq r} n_{j}\left|x_{j}-y_{j}\right| \leq C \sum_{1 \leq j \leq r}\left|x_{j}-y_{j}\right| .
$$

Con los $n_{j}$ positivos y $C=\sum n_{j}$. Dados los conjuntos de generadores $B=$ $\left\{\chi_{1}, \chi_{2}, \ldots, \chi_{r}\right\}, B^{1}=\left\{\delta_{1}, \delta_{2}, \ldots, \delta_{s}\right\}$ de $\Gamma$. Cada $\chi_{i} \in B$ se puede escribir en la forma:

$$
\chi_{i}=\sum_{1 \leq j \leq s} n_{j, i} \delta_{j}, n_{j, i} \in \mathbb{Z}
$$

Entonces

$$
\begin{aligned}
\left|\chi_{i}(x)-\chi_{i}(y)\right| & =\left|\sum_{1 \leq j \leq s}\left(n_{j, i} \delta_{j}\right)(x)-\sum_{1 \leq j \leq s}\left(n_{j, i} \delta_{j}\right)(y)\right| \\
& =\left|\sum_{1 \leq j \leq s}\left[\delta_{j}(x)\right]^{n_{j, i}}-\sum_{1 \leq j \leq s}\left[\delta_{j}(y)\right]^{n_{j, i}}\right|
\end{aligned}
$$

Aplicando la desigualdad (7), tenemos

$$
\left|\chi_{i}(x)-\chi_{i}(y)\right| \leq C_{i} \sum_{1 \leq j \leq s}\left|\delta_{j}(x)-\delta_{j}(y)\right|=C_{i} d_{B^{1}}(x, y)
$$

por lo tanto,

$$
d_{B}(x, y) \leq C^{1} d_{B^{1}}(x, y)
$$


donde $C^{1}=\sum_{i} C_{i}$ no depende de $x, y \in G$. Procediendo de la misma manera, deducimos la existencia de otra constante $C>0$, tal que, para todo $x, y \in G$,

$$
d_{B^{1}}(x, y) \leq C d_{B}(x, y)
$$

De (9) y (10) se concluye que $d_{B}$ es acotadamente equivalente con $d_{B^{1}}$.

3. Ahora se puede ver fácilmente que $d_{B}$ es Lipschitz compatible, pues $d_{B}$ es invariante por (3), además por (8) se concluye que los $\chi_{i}$ están en $\operatorname{Lip}_{1}(G)$ para $i=1, \ldots, r$.

4. Si $d$ es Lipschitz compatible, entonces $\chi_{i} \in \operatorname{Lip}_{1}(G)$ respecto a la métrica $d$ entonces

$$
\left|\chi_{i}(x)-\chi_{i}(y)\right| \leq C d(x, y)
$$

de la desigualdad anterior se sigue la afirmación.

Observe que las métricas

$$
\begin{aligned}
d_{B}(x, y) & :=\sum_{1 \leq i \leq r}\left|\chi_{i}(x)-\chi_{i}(y)\right| \\
d(x, y) & :=\max _{1 \leq i \leq r}\left|\chi_{i}(x)-\chi_{i}(y)\right|
\end{aligned}
$$

son acotadamente equivalentes, pues claramente tenemos,

$$
d(x, y) \leq d_{B}(x, y) \leq r d(x, y)
$$

Además, si $d_{1}, d_{2}, \ldots, d_{r}$ son métrica en $\mathrm{G}$ entonces $\tilde{d}$ definida como,

$$
\tilde{d}(x, y)=d_{1}(x, y)+d_{2}(x, y)+\cdots+d_{r}(x, y)
$$

es una métrica. La métrica $\tilde{d}$ es acotadamente equivalente con la métrica

$$
\hat{d}(x, y)=\max _{1 \leq i \leq r}\left\{d_{i}(x, y)\right\}
$$

También observe que por las propiedades de los $\chi_{i}$ y la invariancia de la métrica $d$, podemos escribir,

$$
\frac{\left|\chi_{i}(x)-\chi_{i}(y)\right|}{d(x, y)}=\frac{\left|\chi_{i}(z)-1\right|}{d(z, e)} \operatorname{con} z=x y^{-1} \in G
$$




\section{Resultado principal}

Una pregunta que nos planteamos es si las métricas Lipschitz compatibles son acotadamente equivalentes a la métrica $d_{B}$, es decir, si todas las métricas Lipschitz compatibles generan el mismo espacio de Lipschitz. El resultado que encontramos es que esto sucede en el grupo $T$, sin embargo, para el grupo $T^{r}$ logramos encontrar condiciones bajo las cuales un grupo de métricas Lipschitz compatibles son acotadamente equivalentes con la métrica $d_{B}$, es decir, generan el mismo espacio de Lipschitz.

Teorema 4 Sea $G$ un grupo topológicamente isomorfo a $T^{r}$ con $r \geq 1$, cuyo dual $\Gamma$ es generado por $B=\left\{\chi_{1}, \chi_{2}, \ldots, \chi_{r}\right\}$. Si d es una métrica Lipschitz compatible en $G$, y para cada $\chi_{i} \in B$ existe una sucesión $\left(x_{n}\right)$ en $G$ tal que $\lim _{n \rightarrow \infty} d\left(x_{n}, e\right)=0$, donde e es el elemento identidad del grupo, y un $0<$ $M<1$ tal que para todo $n \in \mathbb{N}$ se cumple

$$
M \leq \frac{\left|\chi_{i}\left(x_{n+1}\right)-1\right|}{d\left(x_{n}, e\right)} .
$$

Entonces existe una métrica particular $\hat{d}$ del tipo 11 asociada a la métrica d que es acotadamente equivalente a la métrica $d_{B}$.

Demostración. Sea $\chi_{i} \in B$ arbitrario, como $\chi_{i}$ es continuo y la sucesión $\left(x_{n}\right)$ en $G$ satisface $\lim _{n \rightarrow \infty} d\left(x_{n}, e\right)=0$, podemos suponer sin pérdida de generalidad que:

$$
d\left(x_{1}, e\right)>d\left(x_{2}, e\right)>\cdots>d\left(x_{n}, e\right)>d\left(x_{n+1}, e\right)>\ldots
$$

y

$$
\left|\chi_{i}\left(x_{1}\right)-1\right|>\left|\chi_{i}\left(x_{2}\right)-1\right|>\cdots>\left|\chi_{i}\left(x_{n+1}\right)-1\right|>\ldots
$$

Como $d$ es Lipschitz compatible, existe un $N>0$ tal que para cualquier $\chi_{i} \mathrm{y}$ para todo $x \in G$, tenemos

$$
\left|\chi_{i}(x)-1\right| \leq N d(x, e) .
$$

De esto y las desigualdades (12) y (13), se verifica que para cualquier $\chi_{i}$ y todo $n \in \mathbb{N}$

$$
\begin{aligned}
M d\left(x_{n+1}, e\right) & \leq\left|\chi_{i}\left(x_{n+1}\right)-1\right| \\
\left|\chi_{i}\left(x_{n}\right)-1\right| & \leq N d\left(x_{n}, e\right) \\
M d\left(x_{n}, e\right) & \leq\left|\chi_{i}\left(x_{n}\right)-1\right| \\
\left|\chi_{i}\left(x_{n+1}\right)-1\right| & \leq N d\left(x_{n+1}, e\right) \\
M d\left(x_{n}, e\right) & \leq\left|\chi_{i}\left(x_{n+1}\right)-1\right| \leq N d\left(x_{n+1}, e\right) .
\end{aligned}
$$


A partir de las desigualdades (15), (16), 17), (18) y (18) obtenemos,

$$
\frac{M}{N} \leq \frac{d\left(x_{n+1}, e\right)}{d\left(x_{n}, e\right)} \leq \frac{N}{M} \frac{\left|\chi_{i}\left(x_{n+1}\right)-1\right|}{\left|\chi_{i}\left(x_{n}\right)-1\right|} \leq \frac{N^{2}}{M^{2}} \frac{d\left(x_{n+1}, e\right)}{d\left(x_{n}, e\right)},
$$

lo cual implica que para todo $n \in \mathbb{N}$,

$$
\frac{M^{2}}{N^{2}} \leq \frac{\left|\chi_{i}\left(x_{n+1}\right)-1\right|}{\left|\chi_{i}\left(x_{n}\right)-1\right|}
$$

Definimos en $G$, el subgrupo $H_{\chi_{i}}=\left\{x \in G / \chi_{i}(x)=1\right\}$ y consideramos el grupo cociente $G / H_{\chi_{i}}$ de todas las clases laterales, donde

$$
x, y \in H_{\chi_{i}} a \Leftrightarrow \chi_{i}(x)=\chi_{i}(y)=\chi_{i}(a) .
$$

Ahora definimos en el grupo cociente $G / H_{\chi_{i}}$ la métrica $d_{\chi_{i}}^{*}$, como

$$
d_{\chi_{i}}^{*}\left(H_{\chi_{i}} a, H_{\chi_{i}} b\right)=\inf \left\{d(x, y) / x \in H_{\chi_{i}} a \wedge y \in H_{\chi_{i}} b\right\} .
$$

La métrica $d_{\chi_{i}}^{*}$ es una métrica invariante por traslación [2]. Denotamos $H_{\chi_{i}} a=\bar{a}$. Por las propiedades del ínfimo, para todo $u \in \bar{x} \mathrm{y}$ todo $v \in \bar{y}$ se cumple

$$
d_{\chi_{i}}^{*}(\bar{x}, \bar{y}) \leq d(u, v)
$$

entonces $\lim _{n \rightarrow \infty} d_{\chi_{i}}^{*}\left(\bar{x}_{n+1}, \bar{e}\right)=0$. Y además es fácil ver que,

$$
\chi_{i}(\overline{x y})=\chi_{i}(\bar{x}) \chi_{i}(\bar{y}) .
$$

Veamos que la métrica $d_{\chi_{i}}^{*}$ satisface la siguiente propiedad,

$$
\left|\chi_{i}(\bar{x})-1\right|<\left|\chi_{i}(\bar{y})-1\right| \Rightarrow d_{\chi_{i}}^{*}(\bar{x}, \bar{e}) \leq N d_{\chi_{i}}^{*}(\bar{y}, \bar{e}) .
$$

En efecto, considere la siguiente función continua

$$
\begin{array}{r}
f: G \rightarrow \mathbb{R} \\
f(g)=\left|\chi_{i}(g)-1\right| .
\end{array}
$$

En el subconjunto conexo $\bar{B}(e, r)$ de $G$ [5], donde $r=N d_{\chi_{i}}^{*}(\bar{y}, \bar{e})$, se cumple que $f(e) \leq f(x) \leq f(y)$, solo bastaría ver que $f(y) \leq N d_{\chi_{i}}^{*}(\bar{y}, \bar{e})$. Como $d$ es Lipschitz tenemos $\left|\chi_{i}(y)-1\right| \leq N d(y, e)$, usando 20] implicamos que $\left|\chi_{i}(y)-1\right| \leq N d_{\chi_{i}}^{*}(\bar{y}, \bar{e})$, entonces $f(y) \leq N d_{\chi_{i}}^{*}(\bar{y}, \bar{e})$. Por el teorema del valor intermedio existe un $z \in B(e, r)$ tal que $f(z)=f(x)$, es decir, existe un $z \in G$ tal que $d(z, e) \leq N d_{\chi_{i}}^{*}(\bar{y}, \bar{e})$ y $\left|\chi_{i}(z)-1\right|=\left|\chi_{i}(x)-1\right|$, de esta igualdad 
implicamos que $\chi_{i}(z)=\chi_{i}(x)$ o $\chi_{i}(x)=\overline{\chi_{i}(z)}$. En el caso $\chi_{i}(z)=\chi_{i}(x)$ tenemos $d_{\chi_{i}}^{*}(\bar{x}, \bar{e}) \leq d(z, e) \leq N d_{\chi_{i}}^{*}(\bar{y}, \bar{e})$. Para el caso $\chi_{i}(x)=\overline{\chi(z)}$ usamos las igualdades $d(z, e)=d\left(z^{-1}, e\right)$ y $\overline{\chi_{i}(z)}=\chi_{i}\left(z^{-1}\right)$ que son inmediatas, entonces nuevamente por (20) tenemos $d_{\chi_{i}}^{*}(\bar{x}, \bar{e}) \leq d\left(z^{-1}, e\right)=d(z, e) \leq N d_{\chi_{i}}^{*}(\bar{y}, \bar{e})$.

Utilizando el hecho de que el grupo es compacto, podemos afirmar que dado un $\bar{x} \neq \bar{e}$ en $G / H_{\chi_{i}}$, existe un $n \in \mathbb{N}$ tal que

$$
d_{\chi_{i}}^{*}\left(\bar{x}_{n+1}, \bar{e}\right)<N d_{\chi_{i}}^{*}(\bar{x}, \bar{e})<d_{\chi_{i}}^{*}\left(\bar{x}_{n}, \bar{e}\right) .
$$

Usando la contrarrecíproca de (22) tenemos

$$
\left|\chi_{i}\left(\bar{x}_{n+1}\right)-1\right| \leq\left|\chi_{i}(\bar{x})-1\right| .
$$

De aquí, obtenemos

$$
\frac{\left|\chi_{i}\left(\bar{x}_{n+1}\right)-1\right|}{d_{\chi_{i}}^{*}\left(\bar{x}_{n}, \bar{e}\right)} \leq \frac{\left|\chi_{i}(\bar{x})-1\right|}{N d_{\chi_{i}}^{*}(\bar{x}, \bar{e})} .
$$

En el caso que $d_{\chi_{i}}^{*}\left(\bar{x}_{n+1}, \bar{e}\right)<N d_{\chi_{i}}^{*}(\bar{x}, \bar{e})=d_{\chi_{i}}^{*}\left(\bar{x}_{n}, \bar{e}\right)$ implicamos de 22) que $\left|\chi_{i}\left(\bar{x}_{n+1}\right)-1\right| \leq\left|\chi_{i}(\bar{x})-1\right|$, lo que significa que la desigualdad 23) se sigue verificando. Por lo tanto, de 23) podemos deducir que:

$$
\frac{\left|\chi_{i}\left(\bar{x}_{n+1}\right)-1\right|}{d_{\chi_{i}}^{*}\left(\bar{x}_{n+1}, \bar{e}\right)} \frac{d_{\chi_{i}}^{*}\left(\bar{x}_{n+1}, \bar{e}\right)}{d_{\chi_{i}}^{*}\left(\bar{x}_{n}, \bar{e}\right)} \leq \frac{\left|\chi_{i}(\bar{x})-1\right|}{N d_{\chi_{i}}^{*}(\bar{x}, \bar{e})} .
$$

Pero sabemos que $\left|\chi_{i}(x)-1\right| \leq N d(x, e)$ ya que $\chi_{i} \in \operatorname{Lip}_{1}(G)$, además

$$
d_{\chi_{i}}^{*}(\bar{x}, \bar{e})=\inf \left\{d(z, t) / z \in H_{\chi_{i}} x \wedge t \in H_{\chi_{i}} e\right\}
$$

y por las propiedades del ínfimo, para cualquier $x \in G$,

$$
\left|\chi_{i}(x)-1\right| \leq N d_{\chi_{i}}^{*}(\bar{x}, \bar{e})
$$

De (21) y 25] tenemos

$$
d_{\chi_{i}}^{*}\left(\bar{x}_{n}, \bar{e}\right) \leq d\left(x_{n}, e\right)
$$

y

$$
\left|\chi_{i}\left(x_{n+1}\right)-1\right| \leq N d_{\chi_{i}}^{*}\left(\bar{x}_{n+1}, \bar{e}\right)
$$

entonces

$$
\frac{\left|\chi_{i}\left(x_{n+1}\right)-1\right|}{d\left(x_{n}, e\right)} \leq N \frac{d_{\chi_{i}}^{*}\left(\bar{x}_{n+1}, \bar{e}\right)}{d_{\chi_{i}}^{*}\left(\bar{x}_{n}, \bar{e}\right)}
$$


Por otro lado, de la desigualdad (19) tenemos

$$
\frac{M^{2}}{N^{2}}\left|\chi_{i}\left(x_{n}\right)-1\right| \leq\left|\chi_{i}\left(x_{n+1}\right)-1\right|,
$$

de esto y la desigualdad (26), obtenemos

$$
\frac{M^{2}}{N^{2}} \frac{\left|\chi_{i}\left(x_{n}\right)-1\right|}{d\left(x_{n}, e\right)} \leq \frac{\left|\chi_{i}\left(x_{n+1}\right)-1\right|}{d\left(x_{n}, e\right)} \leq N \frac{d_{\chi_{i}}^{*}\left(\bar{x}_{n+1}, \bar{e}\right)}{d_{\chi_{i}}^{*}\left(\bar{x}_{n}, \bar{e}\right)} .
$$

Lo cual implica junto con (17) la siguiente relación

$$
\frac{M^{2}}{N^{2}} M \leq \frac{M^{2}}{N^{2}} \frac{\left|\chi_{i}\left(x_{n}\right)-1\right|}{d\left(x_{n}, e\right)} \leq N \frac{d_{\chi_{i}}^{*}\left(\bar{x}_{n+1}, \bar{e}\right)}{d_{\chi_{i}}^{*}\left(\bar{x}_{n}, \bar{e}\right)}
$$

Es decir,

$$
\frac{M^{3}}{N^{3}} \leq \frac{d_{\chi_{i}}^{*}\left(\bar{x}_{n+1}, \bar{e}\right)}{d_{\chi_{i}}^{*}\left(\bar{x}_{n}, \bar{e}\right)} .
$$

Procediendo de la manera anterior, de las desigualdades

$$
d_{\chi_{i}}^{*}\left(\bar{x}_{n+1}, \bar{e}\right) \leq d\left(x_{n+1}, e\right)
$$

$\mathrm{y}$

$$
\left|\chi_{i}\left(x_{n+1}\right)-1\right|=\left|\chi_{i}\left(\bar{x}_{n+1}\right)-1\right|,
$$

y considerando la desigualdad (15), tenemos

$$
M \leq \frac{\left|\chi_{i}\left(x_{n+1}\right)-1\right|}{d\left(x_{n+1}, e\right)} \leq \frac{\left|\chi_{i}\left(\bar{x}_{n+1}\right)-1\right|}{d_{\chi_{i}}^{*}\left(\bar{x}_{n+1}, \bar{e}\right)} .
$$

Combinando las desigualdades 24, (27) y 28) deducimos que para todo $\bar{x} \neq \bar{e}$ se satisface

$$
\frac{M^{4}}{N^{3}} \leq \frac{\left|\chi_{i}(\bar{x})-1\right|}{N d_{\chi_{i}}^{*}(\bar{x}, \bar{e})} .
$$

En general para todo $\chi_{i} \in \Gamma$ y todo $\bar{x} \neq \bar{e}$, la desigualdad anterior es válida. Por las propiedades de los $\chi_{i}$ y tomando en cuenta que las métricas $d_{\chi_{i}}^{*}$ son invariantes. Entonces para cada $\chi_{i} \in \Gamma$ si $\bar{x}, \bar{y} \in G / H_{\chi_{i}}$. Para $\bar{x} \neq \bar{y}$ se cumple

$$
\frac{M^{4}}{N^{2}} \leq \frac{\left|\chi_{i}(\bar{x})-\chi_{i}(\bar{y})\right|}{d_{\chi_{i}}^{*}(\bar{x}, \bar{y})}
$$


Ahora definimos la métrica $\tilde{d}$ en $G$ como:

$$
\tilde{d}(x, y)=d_{\chi_{1}}^{*}(\bar{x}, \bar{y})+d_{\chi_{2}}^{*}(\bar{x}, \bar{y})+\cdots+d_{\chi_{r}}^{*}(\bar{x}, \bar{y}) .
$$

Verificar que $\tilde{d}$ es una métrica se deduce del hecho de que dado $a \in G, a \neq e$ hay un carácter $\chi \in \Gamma$ tal que $a \neq 1$ [2]. De aquí, si $\tilde{d}(x, y)=0$ entonces $x=y$, las demás propiedades se verifican fácilmente.

La métrica $\tilde{d}$ es acotadamente equivalente con la métrica

$$
\hat{d}(x, y)=\max _{1 \leq i \leq r}\left\{d_{\chi_{i}}^{*}(\bar{x}, \bar{y})\right\}
$$

De las desigualdades 25] y 29] obtenemos para cualquier $x, y \in G$, se verifica

$$
\frac{M^{4}}{N^{2}} \hat{d}(x, y) \leq d_{B}(x, y) \leq N \hat{d}(x, y) .
$$

Corolario $1 \mathrm{Si} G=T$ y d una métrica invariante en $T$. Entonces las siguientes afirmaciones son equivalentes

1. d es una métrica Lipschitz compatible y satisface (12).

2. d es acotadamente equivalente $d_{B}$.

Demostración. Como $G=T$. Entonces el carácter identidad es inyectivo y es un generador de $\Gamma$. De aquí la igualdad 20 implica que $\hat{d}=d$, lo cual implica que $d$ es acotadamente equivalente a $d_{B}$. Inversamente sabemos que $d_{B}$ es Lipschitz compatible y se puede construir una sucesión en $T$ de tal forma que la métrica $d_{B}$ satisface (12). Como $d$ es acotadamente equivalente a $d_{B}$ entonces $d$ es Lipschitz compatible y se comprueba fácilmente que también satisface (12).

\section{Conclusiones}

Si en los espacios topológicos de la forma $T^{r}$ queremos hablar de aproximación trigonométrica generalizada para funciones de Lipschitz, la métrica involucrada en estos espacios debe cumplir con ciertos requisitos, por ejemplo, que los caracteres pertenezcan al espacio de Lipschitz. Es una de las razones por la que trabajamos con métricas llamadas Lipschitz compatibles. Podríamos decir que la métrica $d_{B}$, en cierto sentido, es una métrica canónica, pues en el caso de $T$ cualquier métrica Lipschitz compatibles es acotadamente equivalente a $d_{B}$ lo que significa que generan el mismo espacio topológico y la aproximación se podría 
realizar utilizando $d_{B}$. En el caso de espacios topológicos de la forma $T^{r}$ con $r>1$ encontramos que si cualquier métrica Lipschitz compatible cumple con cierta condición, entonces esta métrica genera una métrica Lipschitz compatible que es acotadamente equivalente a $d_{B}$. Y podríamos hablar en estos espacios topológicos de que la aproximación por polinomios generalizados es posible.

\section{Agradecimientos}

Nuestro agradecimiento a la Benemérita Universidad Autónoma de Puebla. Del mismo modo, queremos reconocer profundamente el trabajo de los revisores de la revista por todas sus observaciones, las cuales hicieron crecer la calidad de nuestro artículo.

\section{Financiamiento}

Los autores no han recibido financiamiento, el artículo ha sido escrito con sus propios recursos.

\section{Referencias}

[1] H. Abels, Reductive groups as metric spaces, in: T.W. Müller (Ed.) Groups: topological, Combinatorial and arithmetic aspects (Bielefeld, 1999), London Mathematical Society Lecture Notes 311, Cambridge University Press, Cambridge, 2004 pp. 1-20. Doi: 10.1017/CBO9780511550706

[2] E. Hewitt, K.A. Ross, Abstract Harmonic Analysis, Vol. I, Structure of Topological Groups, Integration Theory, Group Representations, 2nd Edition, Grundlehren der Mathematischen Wissenschaften 115, Springer,1979. Doi: 10.1007/978-1-4419-8638-2

[3] L. Leindler, A. Meir, V. Totik, On approximation of continuous functions in Lipschitz norms, Acta. Math. Hung. 45(1985), no. 3-4, 441-443. Doi: 10.1007/BF01957041

[4] H. Reiter, J.D. Stegeman, Classical Harmonic Analysis and Locally Compact Groups, Clarendon Press, Oxford, 2000.

[5] W. Rudin, Fourier Analysis on Groups, Interscience, New York NY, 1962.

[6] J. Schiff, S. Shnider, Lie groups and error analysis, Journal of Lie Theory, 11(2001), no. 1, 231-254. 
[7] D. Sherbert, Banach algebras of Lipschitz functions, Pacific J. Math. 13(1963), no. 4, 1387-1399. Doi: 10.2307/1994243

[8] A. Ziv, Relative distance an error measure in round off error analysis, Math. Comp. 39(1982), 563-569. Doi: 10.2307/2007334

[9] N. Weaver, Subalgebras of little Lipschitz algebras, Pacific Journal of Mathematics 173(1996), no. 1, 283-293. 